CERN-TH.6691/93

\title{
On the Determination of the Dilaton-Antisymmetric Tensor Couplings in Supergravity Theories
}

\author{
J. X. $\mathrm{Lu}^{\dagger}$ \\ CERN, Theory Division, CH-1211, Geneva 23, Switzerland \\ jxlu@cernvm.cern.ch
}

\begin{abstract}
A new approach is provided to determine the dilaton-antisymmetric tensor coupling in a supergravity theory by considering the static supersymmetric field configuration around a super extended object, which is consistently formulated in a curved superspace. By this, the corresponding SUSY transformation rules can also be determined for vanishing fermionic fields as well as bosonic fields other than those in the determined coupling. Therefore, we can, in turn, use this determined part of the supergravity theory to study all the related vacuum-like solutions. We have determined the dilaton-antisymmetric tensor couplings, in which each of the antisymmetric tensors is a singlet of the automorphism group of the corresponding superalgebra, for every supergravity multiplet. This actually happens only for $N \leq 2$ supergravity theories, which agrees completely with the spincontent analysis and the classified $N \leq 2$ super $p$-branes, therefore giving more support to the existence of the fundamental Type II $p$-branes. A prediction is made of the $D=$ $9, N=2$ supergravity which has not yet been written down so far.

CERN-TH.6691/93
\end{abstract}

May 1993

$\dagger$ Supported partially by a World Laboratory Fellowship 


\section{Introduction}

Recently, a lot of attention has been given to finding Reissner-Nordström-like and super extended solutions from certain supergravity theories [1, 2, 3, 4, 5, 6, 4, 8,9]. In these solutions, the dilaton-antisymmetric tensor couplings of the corresponding supergravity theories play essential roles. Without this coupling, the dilaton field is as trivial as a scalar field in ordinary general relativity for the aforementioned solutions. Therefore, determining this coupling is essential at least for charged classical solutions. Supergravity multiplets in diverse dimensions have all been classified some time ago (e.g. see [10]). Almost all the corresponding actions or equations of motion have been given either by direct constructions or by dimensional reductions and truncations from $D=11$ or $D=10$ Type IIB supergravity (see e.g. [11]). Therefore, in principle, we should know all these couplings in the corresponding supergravity theories, which are determined by space-time supersymmetries. In this note, we provide a simple approach to determine this coupling from considering the static supersymmetric field configuration produced by a super extended object consistently coupled with background fields, which is exactly opposite to the current direction, i.e. finding super extended solutions from a known supergravity theory. This study is motivated by recent work [9]. The physical motivation here is: if a source is spacetime supersymmetric, what else could the fields around the source be but supersymmetric. From this, we must have a correspondence between a super extended object and a certain supergravity multiplet (possibly reducible), which was also discussed in [12] for some other reasons. Although, unlike in string cases, we are not sure that a super $p$-brane (for $p>1$ ) has its background fields, i.e. the corresponding supergravity multiplet, as its zero modes, classically everything is perfect for formulating the super $p$-brane in a curved superspace, since this only requires that the superspace $(p+2)$-form $H$ and the superspace torsion $T$ satisfy certain constraints [12,13]. These constraints, in general, contain but may not be sufficient to give the field equations of the corresponding supergravity theories, except for the $D=11$ and $D=10, N=1$ cases [12,13]. It is also worth pointing out that as for Green-Schwarz-like super $p$-brane, the space-time supersymmetry and the world-volume supersymmetry are linked by the so-called $\kappa$-symmetry, a local fermionic gauge symmetry. Given a super extended object consistently formulated in a curved superspace, the field 
configuration produced by the object is described by a supersymmetric theory, therefore the dilaton-antisymmetric tensor coupling must be determined from this field configuration. Once this coupling is determined, we can use the corresponding action to find the Reissner-Nordström-like solution. We then find the supersymmetric solution by taking the mass $=$ charge limit since we know that we begin with a supersymmetric theory. Using this supersymmetric solution, we can determine the supersymmetric transformation rules for vanishing gravitino and dilatino. By this approach, we can determine a supergravity theory up to the stage that is good for those vacuum-like solutions related to the determined couplings. It also provides a primary step for determining a complete supergravity theory. Up to present time, only Type I super $p$-brane actions for $p>1$ [12, 13] and superstring actions of both Type I and II are given, although there might exist Type II super $p$-branes for $p>1$ [14]. All these known super $p$-brane actions have manifest both space-time and world-volume supersymmetries, which is crucial to the approach discussed in this paper. In what follows, we use just these supersymmetric actions as examples, to demonstrate that the above is correct. Moreover, our results apply also to those fundamental Type II p-branes and to some other supersymmetric extended solitons classified in [14]. Various implications of this are discussed in detail.

\section{Scaling arguments}

From our experience, we know that the fields involved in a Reissner-Nordström-like or the charge $=$ mass limit extended solution from a supergravity theory are the graviton, the dilaton and an antisymmetric tensor. The most general action for these fields written in canonical variables with standard normalization factors are

$$
I_{D}=\frac{1}{2 \kappa^{2}} \int d^{D} x \sqrt{-g}\left[R-\frac{1}{2}(\partial \phi)^{2}-\frac{1}{2(d+1) !} f(\phi) F_{d+1}^{2}\right]
$$

where $D$ is the space-time dimension, $\kappa$ the $D$-dimensional gravitational constant, $f(\phi)$ the undetermined coupling, and $F_{d+1}$ the field strength of a $d$-form potential $B_{d}$, which is given by

$$
F_{d+1}=d B_{d}
$$


We now consider (2.1) as describing the field configuration produced by a bosonic $(d-1)$-brane source sitting in the same space-time whose world-volume action is

$$
\begin{aligned}
S_{d}=-T_{d} \int d^{d} \xi[ & \frac{1}{2} \sqrt{-\gamma} \gamma^{i j} \partial_{i} X^{M} \partial_{j} X^{N} G_{M N}-\frac{d-2}{2} \sqrt{-\gamma} \\
& \left.+\frac{1}{d !} \epsilon^{i_{1} \cdots i_{d}} \partial_{i_{1}} X^{M_{1}} \cdots \partial_{i_{d}} X^{M_{d}} B_{M_{1} \cdots M_{d}}\right]
\end{aligned}
$$

where $M=0, \cdots, D-1, T_{d}$ the $(d-1)$-brane tension, $G_{M N}$ the $(d-1)$-brane $\sigma$-model metric and $\gamma_{i j}$ the induced world-volume metric, which is given by

$$
\gamma_{i j}=\partial_{i} X^{M} \partial_{j} X^{N} G_{M N}
$$

Actually, (2.3) is the bosonic sector of the corresponding super $p$-brane action, and we postpone the discussion of supersymmetry to the next section. For present purposes, the action (2.3) is enough. We consider the following general scalings of fields $G_{M N}$ and $B_{M_{1} \cdots M_{d}}$ in $(2.3)$

$$
\begin{aligned}
G_{M N} & \rightarrow \lambda^{2} G_{M N} \\
B_{M_{1} \cdots M_{d}} & \rightarrow \beta B_{M_{1} \cdots M_{d}} .
\end{aligned}
$$

Under these scalings, $S_{d} \rightarrow \lambda^{d} S_{d}$ provided we take $\beta=\lambda^{d}$. Hence, $S_{d}$ scales homogeneously. If 2.1 does describe the field configuration, we expect that $I_{D} \rightarrow \lambda^{d} I_{D}$, i.e. scales the same way as $S_{d}$ under the same scalings. From this requirement and the metric relation $G_{M N}=\Omega(\phi) g_{M N}$ as discussed in [15], we have

$$
\begin{aligned}
& f(\phi+c)=\lambda^{-\frac{2 d \tilde{d}}{D-2}} f(\phi), \\
& \Omega(\phi+c)=\lambda^{\frac{2 \tilde{d}}{D-2}} \Omega(\phi),
\end{aligned}
$$

where the constant shift $c$ of the dilaton field corresponds to the rescaling $\lambda$ of the metric and $c=0$ to $\lambda=1$, and where $\tilde{d}=D-2-d$. If there is no dilaton field, i.e. setting $\phi=0$ in the above theory, we should have $f(\phi=0)=1$, which gives the standard field strength kinetic term, and $\Omega(\phi=0)=1$ since now the canonical metric $g_{M N}$ is identical to the $\sigma$-model metric $G_{M N}$. Noticing these facts, it is not difficult to solve the above equations as $\lambda=1+\epsilon$, where $\epsilon$, and therefore $c$, is infinitesimal. The results are

$$
\begin{aligned}
& f(\phi)=e^{-\alpha(d) \phi}, \\
& \Omega(\phi)=e^{\frac{\alpha(d)}{d} \phi},
\end{aligned}
$$


where $\alpha(d)$ is given by

$$
\alpha(d)=\frac{2 d \tilde{d}}{D-2} \frac{\epsilon}{c} .
$$

For future reference, we explicitly list the scalings of the canonical metric $g_{M N}$, the dilaton and the antisymmetric potential $B_{M_{1} M_{2} \cdots M_{d}}$ as

$$
\begin{aligned}
g_{M N} & \rightarrow \lambda^{\frac{2 d}{D-2}} g_{M N}, \\
B_{M_{1} M_{2} \cdots M_{d}} & \rightarrow \lambda^{d} B_{M_{1} M_{2} \cdots M_{d}}, \\
e^{\phi} & \rightarrow \lambda^{\frac{2 d \tilde{d}}{\alpha(d)(D-2)}} e^{\phi} .
\end{aligned}
$$

By simple scaling arguments, we have determined the relation between canonical metric and $\sigma$-model metric, and the action (2.1) as follows

$$
\begin{gathered}
G_{M N}=e^{\frac{\alpha(d)}{d} \phi} g_{M N} \\
I_{D}=\frac{1}{2 \kappa^{2}} \int d^{D} x \sqrt{-g}\left[R-\frac{1}{2}(\partial \phi)^{2}-\frac{1}{2(d+1) !} e^{-\alpha(d) \phi} F_{d+1}^{2}\right] .
\end{gathered}
$$

\section{World-volume approach to super $p$-brane field configurations}

In this section, we will use the world-volume approach to discuss a static super $p$-brane field configuration. Given a super $p$-brane world-volume action formulated consistently in a curved superspace, a static super $p$-brane configuration, which is the most special one, must satisfy the world-volume equations of motion, the supersymmetry transformations, and the superspace constraints on the background fields. The constraints are actually and should be (for the reasons given in section 1) those of certain supergravity theories as formulated in superspace language. In general, they are not sufficient to give all the equations of motion of the corresponding supergravity, except for a very few cases. Even for these cases, extracting the equations of motion from the corresponding superspace constraints is a very complicated process. In what follows, we will demonstrate that a static super $p$-brane field configuration does satisfy the world-volume equations of motion and supersymmetry transformation rules. We are not going to attack the superspace constraints; instead, by a simpler approach, we require that the proposed part (2.11) of 
the bosonic sector of the corresponding supergravity action describe the same super $p$ brane field configuration. By doing this, we determine completely not only the unknown parameter $\alpha(d)$ in (2.8), thus the action (2.11), but also the static field configuration. We will consider, as examples, the only known covariant super $p$-brane actions with manifest $(N=1$ for $p>1$ and $N=1,2$ for $p=1)$ space-time supersymmetries. Certainly, our approach is not limited to these actions, its applicability will be given in section 5 . The Green-Schwarz-like super $(d-1)$-brane action [12,13] is

$$
\begin{aligned}
S_{d}= & -T_{d} \int d^{d} \xi\left(\frac{1}{2} \sqrt{-\gamma} \gamma^{i j} E_{i}{ }^{A} E_{j}{ }^{B} \eta_{A B}-\frac{d-2}{2} \sqrt{-\gamma}\right. \\
& \left.+\frac{1}{d} \epsilon^{i_{1} i_{2} \cdots i_{d}} E_{i_{1}}{ }^{\hat{A}_{1}} E_{i_{2}} \hat{A}_{2} \cdots E_{i_{d}} \hat{A}_{d} B_{\hat{A}_{d} \cdots \hat{A}_{1}}\right)
\end{aligned}
$$

where $\xi^{i}(i=0,1, \cdots, d-1)$ are coordinates for the $d$-dimensional world-volume with metric $\gamma_{i j}$, swept out by the (closed) $(d-1)$-brane in the course of its evolution. The $E_{i} \hat{A}(\hat{A}=A, \alpha)$ are defined by

$$
E_{i}^{\hat{A}}=\partial_{i} z^{\hat{M}} E_{\hat{M}}^{\hat{A}}
$$

where $\partial_{i} \equiv \partial / \partial \xi^{i}, z^{\hat{M}}=\left(X^{M}, \theta\right)$ are the coordinates of $D$-dimensional superspace $(M=$ $0,1, \cdots, D-1)$ and $E_{\hat{M}}{ }^{\hat{A}}$ is the supervielbein. Our metric convention for both $d$ and $D$ dimensions is "mostly plus", i.e. $(-,+,+, \cdots,+)$. Since action (3.1) is a scalar of the super target space, it is invariant under space-time supersymmetry transformation. Besides, it has a very important local fermionic gauge symmetry, the so-called $\kappa$-symmetry, which is, in terms of $\delta z^{\hat{A}} \equiv \delta z^{\hat{M}} E_{\hat{M}} \hat{A}$,

$$
\begin{aligned}
& \delta z^{A}=0, \quad \delta z^{\alpha}=(1+\Gamma)^{\alpha}{ }_{\beta} \kappa^{\beta}(\xi) \\
& \Gamma_{\beta}^{\alpha} \equiv \frac{(-1)^{(d-2)(d+1) / 4}}{d !} \frac{\epsilon^{i_{1} i_{2} \cdots i_{d}}}{\sqrt{-\gamma}} E_{i_{1}}{ }^{A_{1}} \cdots E_{i_{d}}{ }^{A_{d}}\left(\Gamma_{A_{1} \cdots A_{d}}\right)^{\alpha}{ }_{\beta},
\end{aligned}
$$

where the parameter $\kappa^{\beta}$ is a world-volume scalar but space-time spinor, and $\Gamma_{A_{1} \cdots A_{k}}$ is the antisymmetrized product of $k$ (space-time) $\Gamma$-matrices $\Gamma_{A}$. This symmetry, along with the space-time supersymmetry, guarantees the world-volume supersymmetry. Demanding this symmetry gives some constraints on the super background fields, which are actually the superspace constraints of the corresponding supergravity multiplet [13]. For $D=11$ 
or $D=10, N=1$, these constraints are equivalent to the equations of motion of the corresponding supergravity [12,13,16]. The superspace $d$-form potential $B_{d}$ is defined as

$$
B_{d}=\frac{1}{d !} E^{\hat{A}_{1}} \cdots E^{\hat{A}_{d}} B_{\hat{A}_{d} \cdots \hat{A}_{1}}
$$

and its field strength is given by $F_{d+1}=d B_{d}$. The constraint on $F_{d+1}$ requires that it satisfy the superspace Bianchi identity

$$
d F_{d+1}=0
$$

A classification of these super $p$-branes with the allowed $(p, D)$ values was given in [12] for $D-1>p \geq 1$. Besides, the number $N$ of space-time supersymmetries is restricted to 1 for $p>1$, while $N=1,2$ are both possible for $p=1$. These super $p$-branes consist of the four sequences labelled by $\mathbf{R}, \mathbf{C}, \mathbf{H}, \mathbf{O}$ (the four composition-division algebras, for reasons explained in [12]). By inspection one can verify that for each super $p$-brane there is a supergravity multiplet (possibly reducible) containing a $(p+1)$-form gauge field $B$ with a $(p+2)$-form field strength $F=d B$, i.e. the one in (2.11) for $d=p+1$. However, in what follows, we are interested in only $\mathbf{O}$ and $\mathbf{H}$ sequences. The $(p+2)$-form gauge field in sequences $\mathbf{R}$ and $\mathbf{C}$ is either the dilaton itself, or some auxiliary field, or some scalar field in the matter supermultiplet as discussed in [12]. This does not give the coupling that is important for the purpose of this paper, although it may be useful for some other intentions. Actually, it gives $\tilde{d}=D-3-p \leq 0$ and is out of the condition $\tilde{d} \geq 1$ in the following analysis. For $p>1$ the equation of motion for $\gamma_{i j}$ is

$$
\gamma_{i j}=E_{i}{ }^{A} E_{j}{ }^{B} \eta_{A B}
$$

(For $p=1, \gamma_{i j}$ is determined only up to an arbitrary scale factor.) Using this equation one can show that the $\Gamma$ in $(3.3)$ satisfies $\Gamma^{2}=1$, hence $\frac{1}{2}(1+\Gamma)$ is a projection operator. For this reason, only half the components of $\kappa^{\beta}$ are effective in the gauge transformation (3.3) which can be used to gauge away half of the components of $\theta$. After this short review, we are now ready to discuss a static super $(d-1)$-brane field configuration. As usual, we set the fermionic coordinate $\theta=0$, then the super target space is just usual space-time and 
the super $(d-1)$-action (3.1) reduces to its bosonic sector (2.3). In order to have a super $(d-1)$-brane field configuration, we must first have a $d$-dimensional Poincaré symmetry for the space-time metric $G_{M N}$ and the antisymmetric potential $B_{M_{1} \cdots M_{d}}$. We do not necessarily require a transverse $S O(D-d)$ symmetry at the beginning for the metric and the antisymmetric potential, but at the end they do have this symmetry. For simplicity, we impose it from the beginning. As in [9], we make the most general ansatz satisfying the above requirements, for the metric

$$
d S^{2}=e^{2 A(r)} \eta_{\mu \nu} d x^{\mu} d x^{\nu}+e^{2 B(r)} \delta_{m n} d y^{m} d y^{n}
$$

and for the potential

$$
A_{01 \cdots d-1}=-e^{C(r)}
$$

where $\mu=0,1, \cdots, d-1 ; m=d, d+1, \cdots, D-1 ; r=\sqrt{\delta_{m n} y^{m} y^{n}}$ and $A(r), B(r)$ are as yet undetermined $S O(D-d)$ invariant functions. All the other components of $G_{M N}$ and $B_{M_{1} \ldots M_{d}}$ are set to vanish. We choose the static gauge

$$
X^{\mu}=\xi^{\mu}
$$

Since we want to find a super static $(d-1)$-brane configuration, we must have the supersymmetry transformations satisfied for $\theta=0$. This is effectively achieved by requiring the existence of some non-vanishing Killing spinor $\epsilon$ such that

$$
\delta \theta=(1-\Gamma) \epsilon=0
$$

where we have used the local fermionic gauge symmetry (3.3) to gauge away half of the components of $\theta$. By using the static gauge (3.9), (3.7) and (3.6), we have the $\Gamma$ in (3.3),

$$
\Gamma=(-1)^{(d-2)(d+1) / 4} \Gamma_{01 \cdots d-1}
$$

where $\Gamma$ is actually a $d$-dimensional chiral operator as $d$ is even. Equation (3.10) tells that half of the supersymmetries must be broken for this static super $(d-1)$-brane field configuration, which is entirely consistent with what we learned on finding super extended solutions from supergravity theories [1, 2, 3, [4, , 5, 14, 17]. Therefore, we expect that the field 
configuration has a $d$-dimensional super Poincaré symmetry. We now consider the worldvolume equations of motion for vanishing $\theta$. They are actually the same as those derived from the bosonic action (2.3), which are

$$
\begin{aligned}
\partial_{i}\left(\sqrt{-\gamma} \gamma^{i j} \partial_{j} X^{N} G_{M N}\right) & -\frac{1}{2} \sqrt{-\gamma} \gamma^{i j} \partial_{i} X^{N} \partial_{j} X^{P} \partial_{M} G_{N P} \\
& -\frac{1}{d !} \epsilon^{i_{1} \cdots i_{d}} \partial_{i_{1}} X^{N_{1}} \cdots \partial_{i_{d}} X^{N_{d}} F_{M N_{1} \cdots N_{d}}=0
\end{aligned}
$$

and

$$
\gamma_{i j}=\partial_{i} X^{M} \partial_{j} X^{N} G_{M N}
$$

Using the static gauge (3.9) and the ansatz for (3.7) and (3.8), the above equations reduce to

$$
\partial_{m}\left(e^{d A}-e^{C}\right)=0
$$

and

$$
\gamma_{\mu \nu}=e^{2 A} \eta_{\mu \nu}
$$

Equation (3.14) is the so-called "no-force" condition, and the term within the bracket is actually the potential (see [2,3]). Hence eq. (3.14) implies that the potential is constant, and the $(d-1)$-brane itself therefore does not feel any back-reaction. We can always choose the zero point of the potential so that the constant vanishes. Thus, we have from (3.14),

$$
C=d A
$$

In the next section, we will determine the unknown parameter $\alpha(d)$, and the functions $A$ and $B$.

\section{Determination of the couplings}

In [9], we found the $(d-1)$-brane field configuration by imposing $P_{d} \times S O(D-d)$ symmetry on the background fields simply from the combined bosonic action (2.11) + (2.3). The surprising thing is that the unknown parameter $\alpha(d)$ is determined in the process of finding solutions for $D>d+2$, where $D$ the space-time dimension. We are not going to repeat the same derivation here. Instead, we just quote the solitonic $(d-1)$-brane 
solution for an arbitrary $\alpha(d)$ from [9], and demand that this solitonic configuration for $r \neq 0$ satisfy the world-volume equations of motion, i.e. the "no-force" condition discussed in the previous section, then find the same $\alpha(d)$. This is analogous to dealing with Dirac's monopole, which can be described either by a singular Dirac-string in a space with trivial topology or by $\mathrm{Wu}$-Yang construction in a space with non-trivial topology. For $r \neq 0$, both descriptions give the same field configuration. Things here are a little different. We do not need a source to find a magnetic or topological $(d-1)$-brane solution from the equations of motion of the dual action of (2.11). This dual action is

$$
\tilde{I}_{D}=\frac{1}{2 \kappa^{2}} \int d^{D} x \sqrt{-g}\left[R-\frac{1}{2}(\partial \phi)^{2}-\frac{1}{2(\tilde{d}+1) !} e^{-\alpha(\tilde{d}) \phi} \tilde{F}_{\tilde{d}+1}^{2}\right]
$$

where $\alpha(\tilde{d})=-\alpha(d)$ and

$$
\tilde{F}_{\tilde{d}+1}=e^{-\alpha(d) \phi *} F_{d+1},
$$

where ${ }^{*}$ is the Hodge dual operator. We can find solitonic $(d-1)$-brane solutions from (4.1) for an arbitrary $\alpha(d)$. However, the Dirac-string-like, so-called elementary solutions are obtained in [9] only for fixed $\alpha(d)$. The reason is that the sources are extended objects, described by actions (2.3), some of which, i.e. those in $\mathbf{O}$ and $\mathbf{H}$ sequences, are actually supersymmetric. This is one example to show that extended objects differ from point particles. We expect that if we require that the solitonic configurations for an arbitrary $\alpha(d)$ satisfy the world-volume equations of motion, i.e. the "no-force" condition discussed in the previous section, we can also fix the $\alpha(d)$. This is indeed the case. We are about to demonstrate it explicitly. As before, the most general ansatz for the metric with $P_{d} \times$ $S O(D-d)$ symmetry is

$$
d s^{2}=e^{2 a(r)} \eta_{\mu \nu} d x^{\mu} d x^{\nu}+e^{2 b(r)} \delta_{m n} d y^{m} d y^{n}
$$

where notations have the same meanings as those in (3.7), except that $d s^{2}$ is a canonical metric. The relations between $d s^{2}, a$ and $b$ and $d S^{2}, A$ and $B$ are given, by using (2.10), as

$$
\begin{aligned}
d S^{2} & =e^{\frac{\alpha(d)}{d} \phi} d s^{2}, \\
A & =a+\frac{\alpha(d)}{2 d}, \\
B & =b+\frac{\alpha(d)}{2 d} .
\end{aligned}
$$


The solitonic $(d-1)$-brane solution for an arbitrary $\alpha(d)$ from (4.1) is

$$
\begin{aligned}
& \tilde{F}_{\tilde{d}+1}= Q \epsilon_{\tilde{d}+1}, \\
& d s^{2}= {\left[1+\frac{|Q|}{\tilde{d} r \tilde{d}}\right]^{\frac{-\tilde{d}}{D-2}} \eta_{\mu \nu} d x^{\mu} d x^{\nu} } \\
&+\left[1+\frac{|Q|}{\tilde{d} r^{\tilde{d}}}\right]^{\frac{d}{D-2}}\left(d r^{2}+r^{2} d \Omega_{\tilde{d}+1}^{2}\right), \\
& e^{-\frac{2}{\alpha(d)} \phi}=1+\frac{|Q|}{\tilde{d} r \tilde{d}},
\end{aligned}
$$

where we have set $\phi_{0}=0$ and $\epsilon_{n}$ is the volume element of the unit $n$-sphere. By using (4.5) and the dual relation (4.2), we can read the $(d+1)$-form $F_{d+1}$ as

$$
F_{r 01 \cdots d-1}=-\frac{2}{\alpha} e^{\left(\alpha-\frac{2}{\alpha}+\frac{2 d \tilde{d}}{\alpha(D-2)}\right) \phi} \partial_{r} \phi .
$$

By using the "no-force" condition (3.16), the relation (4.4) and the metric $g_{M N}$ in (4.5), we can read the field strength (2.2) of potential $B_{d}$ as,

$$
\begin{aligned}
F_{r o 1 \cdots d-1} & =-\partial_{r} e^{C} \\
& =-\left(\frac{\alpha}{2}+\frac{d \tilde{d}}{\alpha(D-2)}\right) e^{\left(\frac{\alpha}{2}+\frac{d \tilde{d}}{\alpha(D-2)}\right) \phi} \partial_{r} \phi
\end{aligned}
$$

Identifying eq. (4.6) with (4.7) for $r \neq 0$, we have

$$
\frac{2}{\alpha(d)}=\frac{\alpha(d)}{2}+\frac{d \tilde{d}}{\alpha(d)(D-2)} .
$$

Solving this simple equation, we have

$$
\alpha^{2}(d)=4-\frac{2 d \tilde{d}}{D-2},
$$

which was already obtained in [9] by finding $(d-1)$-brane solutions from $(2.11)+(2.3)$. We expect that these $\alpha(d)$ for $d \geq 2$ give the correct couplings in the corresponding $N=1$ supergravity theories, whose $(d, D)$ fall in either $\mathbf{O}$ or $\mathbf{H}$ sequences. By inspection, this is indeed the case. These couplings actually cover all the dilaton-antisymmetric tensor ones except for those containing 2-forms in $N=1$ supergravity theories. We determine the $\alpha(d)$ simply by demanding the solitonic solution with an arbitrary $\alpha(d)$ satisfying the worldvolume equations of motion, i.e. the "no-force" condition. Therefore, it is determined by 
space-time supersymmetry, since the static configuration in the previous section is supersymmetric. One may ask why those $\alpha(1)$ for $d=1$ in the same sequences do not describe the right couplings, involving 2 -forms in the corresponding supergravity theories. The simple answer is that we do not have equal on-shell matching of fermionic and bosonic degrees of freedom for the superparticle, which implies no world-line supersymmetry, although we can have both space-time supersymmetry and local $\kappa$-gauge symmetry. In order to obtain the correct coupling, the crucial thing is that we must have equal on-shell fermionic and bosonic degrees of freedom for our field configuration, which can be achieved by having the $\kappa$-symmetry for a $p \geq 1$ extended object with space-time supersymmetries. However, this is not the whole story, we will come back to this point later on. Does our $\alpha(d)$ apply to $N \geq 2$ supergravity theories? If so, what does this imply? This would be the topic of the next section.

\section{On $N \geq 2$ super extended objects and supergravity theories}

By inspection, our $\alpha(d)$ for $d>1$ also give the correct couplings in Type II $D=$ 10 supergravity theories. This is also true whenever we have a coupling containing an antisymmetric tensor as a singlet of the internal symmetry of any given $D \leq 9, N \geq 2$ supergravity multiplet. Careful inspection shows that this actually happens only for $N=2$ supergravity theories. This is entirely consistent with the spin-content analysis, given in [4], that we cannot go beyond spin 1 on the world-volume; therefore we cannot go beyond Type II super extended objects. These results are quite unexpected. From our previous discussions, the immediate conclusion we can draw is that the corresponding $N=2$ super $p$-brane actions, if they exist at all, must reduce to the bosonic $p$-brane actions (2.3) after we set the fermionic fields and possible world-volume spin 1 fields to vanish. At the same time, we break only half of the space-time supersymmetries. We may also jump to the conclusion that there might exist supercovariant actions with $N=2$ space-time supersymmetries for

all the aforementioned cases. This is possibly true for only Type II $D=10 p$-branes for $p \geq 0$. We cannot have a supercovariant action with a world-volume vector field for each of $N=2, D \leq 9$ cases, since now there is no equal on-shell matching of bosonic and fermionic degrees of freedom, as discussed in [14]. Our results not only support the 
spin-content analysis but also provide a first step toward constructing the complete Type II super $p$-brane actions for $p>1$. But we still have quite a few unanswered questions on those lower-dimensional supersymmetric soliton solutions obtained from dimensional and double-dimensional reductions of higher-dimensional solutions. It is actually the main topic of this section to understand the nature of all these solutions. The first question is to know why our approach applies only to those aforementioned cases, and not to all the soliton solutions classified in [9, 14]. For concreteness, we take $N=2, D=8$ supergravity [18] as an example. Its field content is

$$
g_{M N}, \quad 2 \psi_{M}, \quad 6 \chi, \quad A_{M N P}, \quad 3 A_{M N}, \quad 6 A_{M}, \quad 7 \phi
$$

where spinors are pseudo-Majorana and the bosons are real. The automorphism group of the superalgebra is $S U(2)$. With respect to this $S U(2), \psi_{\mu}^{a}(a=1,2)$ is a 2-spinor, $\chi_{i}^{a}$ is a vector-spinor, $A_{\mu \nu}^{i}$ is a triplet, and the vectors split into two triplets. Besides, there is another internal-symmetry global $S L(3, R) \times \operatorname{local} S O(3)$, and the down and up indices $i$ are also in the representations 3 and $\overline{3}$ of global $S L(3, R)$, respectively. The seven scalars parametrize the coset $S L(3, R) / S O(3)$ and transform as $5+1+1$ under the $S U(2)$. All the supersymmetric solutions of Type IIA $D=10$ supergravity, which are independent of more than one spatial dimension, must also be those of the supergravity defined above, since the latter can be obtained from the former by a dimensional reduction on $S^{1} \times S^{1}$. As discussed in [9], any solution with the bosonic symmetry $P_{d} \times S O(D-$ $n) \times\left(S^{1}\right)^{n-d}(D-3 \geq n \geq d)$ and breaking half of the space-time supersymmetries guarantees solutions with bosonic symmetries $P_{k} \times S O(D-l) \times\left(S^{1}\right)^{l-k}$ with $d \geq k>0$ and $n \leq l \leq D-3$ and also breaking half of the space-time supersymmetries. Therefore, we should have six identical supersymmetric-particle solutions corresponding to six 1-form potentials, three identical string solutions to three 2-form potentials, and one membrane to one 3 -form potential in the supermultiplet. We have the same number of solutions for the dual object of each of the above cases. All these solutions break half of the space-time supersymmetries and have the corresponding maximal bosonic symmetries: $P_{1} \times S O(7)$ for superparticle, $P_{2} \times S O(6)$ for superstring, $\cdots$, and $P_{5} \times S O(3)$ for super 4-brane. However, these solutions have no fundamental world-volume actions but gauge-fixed ones 
by dimensional and double-dimensional reductions of Type II $D=10$ super $p$-branes. Each of these solutions guarantees those with smaller bosonic symmetries, as described above. Out of these solutions, our approach applies only to the membrane solutions since only the 3 -form is a singlet of the internal symmetry. Our $\alpha(d)$ for $d=3, D=8$ indeed gives the correct coupling. Let us explain it in detail and take the 2 -forms $A_{M N}^{i}$ as examples. We have three string solutions with the maximal bosonic symmetry $P_{2} \times S O(6)$, one of which comes from a Type IIA $D=10$ superstring by dimensional reduction on $S^{1} \times S^{1}$, and the other two come from a Type IIA supermembrane by different routes. One route is first to dimensionally reduce the $D=10$ membrane to $D=9$, then to $D=8$ by doubledimensional reduction. The other switches just the order of dimensional and doubledimensional reductions. From the $D=10$ point of view, a Type II string in $D=8$ is either a string or a membrane in $D=10$. Therefore, we should not expect to find simultaneously all three strings preserving the maximal bosonic symmetry and breaking just half of space-time supersymmetries: were it the case, we would find simultaneously a Type IIA superstring and two Type IIA supermembranes in $D=10$, but just breaking half of the space-time supersymmetries. It is obviously impossible. So we can find at most two strings at one time from $D=8, N=2$ supergravity. Inspecting the equations of motion and the SUSY transformation rules, we indeed find the case. All these imply that if we want to find string solutions breaking just half of the space-time supersymmetries, we must pick a specific direction of the internal symmetry, i.e. break the internal symmetry down to global $U(1) \times$ global $S L(2, R) \times \operatorname{local} S O(2)$. This in turn implies that the $D=10$ dilaton field for the string solution is not a singlet of the internal symmetries of $D=8, N=2$ supergravity. Therefore, our approach does not apply to this kind of solutions since the space-time metric $g_{M N}$ in a given dimension $D$ is always a singlet of the internal symmetry of a given supergravity theory*. However, the membrane solution has nothing to do with the internal symmetry, the $D=10$ dilaton can now be identified with the $D=8$ one, and our approach works. Indeed, this is the general feature of our approach in all supergravity

* More precisely, our discussion in section 2 does not apply to this kind of solution, but our approach may still do. 
theories, and explains why it always works, except for 2-form field strength in each of Type I cases.

We conclude that our approach always gives the correct coupling if the corresponding antisymmetric gauge potential is a singlet of the internal symmetry of a certain supergravity theory and there exists either a fundamental or a gauge-fixed super $p$-brane action ( $p \geq 1)$ consistently coupled with this potential.

We now come to talk about superparticles. As discussed in the previous section, our approach does not, in general, apply to these. However, one may notice already that our $\alpha(d)$ does give the correct coupling for $d=1$ in Type IIA $D=10$ supergravity. The determination of this $\alpha(1)$ is actually not through the superparticle, but its dual object, a super 6-brane for which we have equal on-shell matching of bosonic and fermionic degrees of freedom. Hence besides those conditions mentioned above for our approach, if there exists a super $p$-brane with $p \geq 1$ and the field strength of its antisymmetric background field is dual to a 2 -form, then the $\alpha$ associated with the 2 -form is correctly determined and must be with opposite sign to that of the $p$-brane. At the same time, we know that there exists also a superparticle solution from the corresponding supergravity. By inspection, our $\alpha(d)$ for $d=1$ also give correct couplings for $N=2, D=9$ and $N=2, D=5$ supergravity theories. This suggests that we might have $N=2$ superparticles in these dimensions. By counting, we do have equal on-shell matching of fermionic and bosonic degrees of freedom for these superparticles. Further investigations show that equal on-shell matching of bosonic and fermionic degrees of freedom happens also for $N=2$ superparticles in $D=3$ and $D=2$. These superparticles, if they exist at all, must share all the properties of Type I super $p$-branes. Therefore, we expect that they can be obtained from $N=2$ superstrings by double-dimensional reductions described in [16], and their world-volume actions are just those given in [13] for $p=0$.

We have one last thing to say in this section, i.e. that our above discussion does not apply to self-dual cases, since we did not incorporate anything on self-dual properties. Thus we should not expect that our $\alpha(d)$ gives the right couplings for those cases. As we know, there exist only two cases, and only for Type II supergravity theories: one is $D=10$ Type IIB supergravity with a self-dual 5 -form, the other is $D=6$ pure Type IIB 
supergravity with a self-dual 3-form. The former one should correspond to a self-dual Type IIB super 3-brane [5], and the latter to a self-dual Type IIB superstring [17]. The bosonic sector of the self-dual Type IIB superstring should also be given by (2.3), but with an additional self-dual relation for the 3 -form. Our previous discussions tell that the bosonic part of Type II super $p$-brane, after setting zero fermionic coordinates and world-volume spin 1 field, is also given by (2.3). Therefore, the same situation as for the self-dual string may apply to the self-dual Type IIB super 3-brane. It is very easy to get the answers. In both cases, we should no longer use the action (2.11) because of the self-dual relations for 3 -form and 5-form. However, the equations of motion derived for graviton and dilaton are still valid. For the self-dual string, these equations should be identical, whether we use $F_{3}$ or ${ }^{*} F_{3}$. More precisely, the $\alpha$ should be the same for either $F_{3}$ or ${ }^{*} F_{3}$. This immediately leads to $\alpha(3)=-\alpha(3)$, i.e. $\alpha(3)=0$. The same applies to the 5 -form, so $\alpha(5)=0$. Hence for self-dual cases, $\alpha(d)=0$ even without referring to solutions. It is worth pointing out that $\alpha(5)=0$ also fits into eq. (4.8). We do not know if there is a deep physical reason behind this.

\section{Space-time supersymmetry}

Once the action (2.11) is determined, we can use it to find Reissner-Nordström-like solutions. Since we know that the action (2.11) is part of the bosonic sector of some supergravity action for each $\alpha$, the charge = mass limit of the corresponding ReissnerNordström-like solution must be the supersymmetric configuration that solves both the equations of motion and the supersymmetric transformation rules for vanishing gravitino $\psi_{M}$ and dilatino $\lambda$, of the corresponding supergravity theory. We will use this fact to determine the supersymmetric transformation rules for vanishing $\psi_{M}$ and $\lambda$. After doing this, we have reached a stage in the supergravity action, which is good for studying almost all the vacuum-like solutions of the supergravity theory that are related to the determined couplings. The general Reissner-Nordström-like extended solutions of (2.11), in diverse dimensions, have been given in [9] for the $\alpha(d)$ given by (4.9). Their charge $=$ mass limits 
have also been given there; they are quoted as:

$$
\begin{array}{ll}
a=\frac{\tilde{d}}{2(D-2)} C, & b=-\frac{d}{2(D-2)} C, \\
B_{01 \cdots d-1}=-e^{C}, & \phi=\frac{\alpha(d)}{2} C,
\end{array}
$$

and

$$
e^{-C}=1+\frac{|Q|}{\tilde{d} r \tilde{d}}
$$

where $D$ is the space-time dimension, $a$ and $b$ are defined by (4.3), and we have set $C_{0}=0$ above. Since fermionic fields differ for different supergravity multiplets, and could be Dirac, Majorana, Weyl or Majorana-Weyl, etc., the most general supersymmetric transformation rule for a given spinor depends crucially on its kind. Hence we have to deal with each supergravity multiplet separately. In what follows, we just give a familiar example, i.e. the $D=10, N=1$ supergravity with 3 -form field strength, to demonstrate the procedure. For this supergravity, both gravitino and dilatino are Majorana-Weyl, but with opposite chirality. The most general supersymmetric transformation rules for vanishing $\psi_{M}$ and $\lambda$ are

$$
\begin{aligned}
\delta \lambda & =\Gamma^{M} \partial_{M} \phi \epsilon+g(\phi) \Gamma^{M N P} F_{M N P} \epsilon=0, \\
\delta \psi_{M} & =D_{M} \epsilon+h(\phi)\left(\Gamma_{M}{ }^{N P Q}-t \delta_{M}{ }^{N} \Gamma^{P Q}\right) F_{N P Q} \epsilon=0,
\end{aligned}
$$

where we have dropped the overall factors and set the $D=10$ gravitational constant $\kappa=1 ; g(\phi)$ and $h(\phi)$ are two as yet undetermined functions of the dilaton field, $t$ is an undetermined numerical constant, and $\epsilon$ is the $D=10$ Majorana-Weyl spinoral parameter, which satisfies

$$
\Gamma_{11} \epsilon=\Gamma_{11}
$$

where $\Gamma_{11}$ is defined as

$$
\Gamma_{11} \equiv \Gamma_{0} \Gamma_{1} \cdots \Gamma_{9}
$$

with flat indices $0,1, \cdots, 9$. We first use the scaling arguments described in section 2 to determine the functions $g(\phi)$ and $h(\phi)$. We expect that each of the equations in $(6.3)$ should scale homogeneously under the following scalings

$$
\begin{aligned}
g_{M N} & \rightarrow \lambda^{1 / 2} g_{M N}, \\
e^{\phi} & \rightarrow \lambda^{3 / \alpha(2)} e^{\phi}, \\
B_{M N} & \rightarrow \lambda^{2} B_{M N},
\end{aligned}
$$


which are obtained by setting $D=10, d=2$ from the general scalings (2.9) in section 2 . Since the $\alpha(d)$ is determined up to its square, however, we can always choose the sign for the $\alpha(d)$ if the considered supergravity multiplet involves only one antisymmetric tensor $F_{d+1}$. The reason is that we can always get the chosen sign for the $\alpha(d)$ by sending $\phi \rightarrow-\phi$ in the action (2.11). But for supergravity involving more than one antisymmetric tensor, we must take care of the relative signs of those $\alpha$ 's. This will be the topic of the next section. In what follows, we simply choose the positive sign, i.e. from (4.9), $\alpha(2)=1$. By noting that $\Gamma_{M} \rightarrow \lambda^{\frac{1}{4}} \Gamma_{M}$ in (6.3), it is easy to know that $g(\phi)$ and $h(\phi)$ must scale as

$$
\begin{aligned}
& g(\phi) \rightarrow \lambda^{-3 / 2} g(\phi), \\
& h(\phi) \rightarrow \lambda^{-3 / 2} h(\phi) .
\end{aligned}
$$

Comparing with the scaling of $e^{\phi}$ in $(\sqrt[6.6]{6)}$, we have

$$
\begin{aligned}
& g(\phi)=g_{0} e^{-\phi / 2}, \\
& h(\phi)=h_{0} e^{-\phi / 2},
\end{aligned}
$$

where $g_{0}$ and $h_{0}$ are as yet two undetermined constants. We proceed by substituting (6.1) for $D=10, d=2$ to $(6.3)$. The results are

$$
\begin{aligned}
\delta \lambda= & \frac{1}{2} \Gamma^{m} \partial_{m} C\left(1+2 \cdot 3 ! g_{0} \Gamma_{01}\right) \epsilon=0, \\
\delta \psi_{\mu}= & \partial_{\mu} \epsilon-\frac{3}{16} \Gamma_{\mu}^{n} \partial_{n} C\left(1-\frac{32}{3} t \cdot h_{0} \Gamma_{01}\right) \epsilon=0, \\
\delta \psi_{m}= & \partial_{m} \epsilon-2 t \cdot h_{0} \Gamma_{01} \partial_{m} C \\
& +\frac{1}{16} \partial_{n} C \Gamma_{m}^{n}\left(1-16 \cdot 3 ! h_{0} \Gamma_{01}\right) \epsilon=0,
\end{aligned}
$$

where all indices are world ones except for the flat 0,1 in the $\Gamma_{01}$. By (3.10) and (3.11), as discussed in section 3 from the world-volume point of view, the supersymmetric configuration (6.1) must break half of the space-time supersymmetries. For the present case, it is equivalent to saying

$$
\left(1-\Gamma_{01}\right) \epsilon=0
$$

where indices 0 and 1 are flat, too. Combining (6.9) with (6.10), we have

$$
g_{0}=-\frac{1}{12}, \quad h_{0}=\frac{1}{96}, \quad t=9
$$


Inserting the above $g_{0}, h_{0}$ and $t$ back into (6.3), the resulting transformation rules for vanishing $\psi_{M}$ and $\lambda$ agree perfectly with their correspondents derived either by direct construction or by dimensional reduction and truncation from $D=11$ supergravity.

\section{Cases involving many antisymmetric tensors}

For a supergravity multiplet involving many antisymmetric tensors, we first determine the action and supersymmetric transformation rules for each of the antisymmetric tensors as described in the previous sections. Then, the action and supersymmetric transformation rules involving many antisymmetric tensors are easily obtained: the single antisymmetric tensor contribution in (2.11) and the corresponding supersymmetric transformation rules are replaced by the contributions from all antisymmetric tensors. In this way, we have constructed a supergravity action, and its SUSY transformation rules, for vanishing fermionic fields, up to such a stage that it is useful for almost all the vacuum-like solutions of the full supergravity theory. However, we still have one unsolved problem, as mentioned earlier, i.e. we do not know how to determine the relative signs of those $\alpha$ 's in both the action and SUSY transformation rules. Actually, there exist only two such supermultiplets to which our approach applies, and both of them live in $D=10$ : one is Type IIA supergravity, the

other Type IIB. We do not have any problem with the Type IIB one, since we have only two $\alpha$ 's and one is zero. So we need to deal with the Type IIA one only. Up to relative signs of the $\alpha$ 's, the determined Type IIA action and supersymmetric transformation rules for vanishing gravitino $\psi_{M}$ and dilatino $\lambda$ are

$$
\begin{aligned}
I_{10}(\text { Type } \quad \text { IIA })= & \frac{1}{2 \kappa^{2}} \int d^{10} x \sqrt{-g}\left[R-\frac{1}{2}(\partial \phi)^{2}-\frac{1}{2 \cdot 2 !} e^{-\alpha(1) \phi} F_{2}^{2}\right. \\
& \left.-\frac{1}{2 \cdot 3 !} e^{-\alpha(2) \phi} F_{3}^{2}-\frac{1}{4 !} e^{-\alpha(3) \phi} F_{4}^{2}\right]
\end{aligned}
$$

and

$$
\begin{aligned}
\delta \lambda= & \Gamma^{M} \phi \Gamma^{11} \epsilon-\frac{\alpha(1)}{4} e^{-\alpha(1) / 2} \Gamma^{N P} F_{N P} \epsilon \\
& +\frac{i \alpha(2)}{12} e^{\frac{-\alpha(2)}{2}} \Gamma^{N P Q} F_{N P Q} \epsilon \\
& +\frac{i \alpha(3)}{48} e^{-\alpha(3) / 2} \Gamma^{N P Q R} F_{N P Q R} \epsilon,
\end{aligned}
$$




$$
\begin{aligned}
\delta \psi_{M}= & D_{M} \epsilon+\frac{1}{64} e^{-\alpha(1) / 2}\left(\Gamma_{M}{ }^{N P}-14 \delta_{M}{ }^{N} \Gamma^{P}\right) F_{N P} \Gamma^{11} \epsilon \\
& +\frac{1}{96} e^{-\alpha(2) / 2}\left(\Gamma_{M}{ }^{N Q}-9 \delta_{M}{ }^{N} \Gamma^{P Q}\right) F_{N P Q} \Gamma^{11} \epsilon \\
& +\frac{i}{256} e^{-\alpha(3) / 2}\left(\Gamma_{M}{ }^{N P Q R}-\frac{20}{3} \delta_{M}{ }^{N} \Gamma^{P Q R}\right) F_{N P Q R} \epsilon
\end{aligned}
$$

where, from (4.9),

$$
\alpha^{2}(1)=\frac{9}{4}, \quad \alpha^{2}(2)=1, \quad \alpha^{2}(3)=\frac{1}{4},
$$

where $\Gamma^{M}$ are the $D=10$ Dirac matrices, where the covariant derivative is given by

$$
D_{M}=\partial_{M}+\frac{1}{4} \omega_{M A B} \Gamma^{A B}
$$

with $\omega_{M A B}$ the Lorentz spin connection, where

$$
\Gamma^{N_{1} N_{2} \cdots N_{n}}=\Gamma^{\left[N_{1}\right.} \Gamma^{N_{2}} \cdots \Gamma^{\left.N_{n}\right]}
$$

and where

$$
\Gamma^{11}=i \Gamma^{0} \Gamma^{1} \cdots \Gamma^{9}
$$

with flat indices $0,1, \cdots, 9$. It seems that our approach does not provide a way to determine the relative signs of the $\alpha$ 's in (7.4). Here we appeal to string quantum-loop arguments. We know that this supergravity is the field theory limit of Type IIA superstring. If we write the action (7.1) in string $\sigma$-model variables, each term in the action should correspond to a positive or at least a tree-level string quantum loop, because we have a good superstring quantum theory. Since the overall sign for those $\alpha$ 's is not important classically, for simplicity, we choose $\alpha(2)=1$ from $\alpha^{2}(2)=1$. Writing the action (7.1) in string $\sigma$-model variables, i.e. from (2.10) and (4.9) for $d=2, D=10$,

$$
G_{M N}=e^{\phi / 2} g_{M N}
$$

we have

$$
\begin{aligned}
I_{10}(\text { Type IIA })= & \frac{1}{2 \kappa^{2}} \int d^{10} x \sqrt{-G} e^{-2 \phi}\left[R+4(\partial \phi)^{2}-\frac{1}{2 \cdot 2 !} e^{\frac{1-2 \alpha(1)}{2} \phi} F_{2}^{2}\right. \\
& \left.-\frac{1}{2 \cdot 3 !} F_{3}^{2}-\frac{1}{4 !} e^{\frac{3-2 \alpha(3)}{2} \phi} F_{4}^{2}\right]
\end{aligned}
$$


where we can read the string loop-counting parameter as $e^{\phi}$. Hence both $1-2 \alpha(1) / 4$ and $3-2 \alpha(3) / 4$ must be integers $\geq 0$. From (7.4), this is only possible for $\alpha(1)=-3 / 2$ and $\alpha(3)=-1 / 2$. Inserting those $\alpha$ 's back into (7.1), (7.2) and (7.3), we get almost the same as those obtained by dimensional reduction from $D=11$ supergravity with vanishing fermionic fields. Our approach does not fix the $W Z$ term and those modified terms in $F_{2}, F_{3}$ and $F_{4}$ in the Type IIA bosonic action, since they vanish for our static configurations.

\section{A prediction of $D=9, N=2$ supergravity}

The $D=9, N=2$ supergravity has field content

$$
g_{M N}, \quad 2 \psi_{M}, \quad A_{M N P}, \quad 2 B_{M N}, \quad 3 A_{M}, \quad 4 \chi, \quad 3 \phi
$$

The three scalars parametrize the coset $G L(2, R) / S O(2)$. The spinors are pseudoMajorana. This theory has not been written down so far because it is tedious, even though trivial, to obtain by dimensional reduction from $D=11$ supergravity. Since the 3 -form potential $A_{M N P}$ is a singlet of internal symmetry $G L(2, R) \times S O(2)$, and the corresponding supermembrane action must be the gauge-fixed one of the $D=11$ supermembrane, we should be able to determine, by our approach, the corresponding coupling and supersymmetric transformation rules for vanishing fermionic fields. From our formulae (4.9) and (2.11), the coupling should be

$$
I_{9}(N=2)=\frac{1}{2 \kappa^{2}} \int d^{9} x \sqrt{-g}\left[R-\frac{1}{2}(\partial \phi)^{2}-\frac{1}{2 \cdot 3 !} e^{-\frac{2}{\sqrt{7}} \phi} F_{4}^{2}\right],
$$

where $F_{4}=d A_{3}$. The determined SUSY transformation rules for vanishing fermionic fields and bosonic fields other than those in (8.2) are

$$
\begin{aligned}
\delta \chi_{i} & =\Gamma_{i} \Gamma^{M} \partial_{M} \phi \epsilon+\frac{1}{4 ! \cdot \sqrt{7}} e^{-\frac{\phi}{\sqrt{7}}} \Gamma_{i} \Gamma^{N P Q R} F_{N P Q R} \epsilon \\
\delta \psi_{M} & =D_{M} \epsilon-\frac{1}{224} e^{-\frac{\phi}{\sqrt{7}}}\left(\Gamma_{M}{ }^{N P Q R}-\frac{16}{3} \delta_{M}{ }^{N} \Gamma^{P Q R}\right) F_{N P Q R} \epsilon
\end{aligned}
$$

where $M=0,1, \cdots, 8$ and $i=1,2$ are $S O(2)$ vector indices, where $D_{M}$ is given by

$$
D_{M}=\partial_{M}+\frac{1}{4} \omega_{M A B} \Gamma^{A B}
$$


with $\omega$ the Lorentz spin connection, and where $\Gamma^{i}$ and $\Gamma^{A}$ are given by

$$
\Gamma^{i}=1 \times \sigma^{i}, \quad \Gamma^{A}=\gamma^{A} \times \sigma^{3},
$$

with $\gamma^{A}$ the $D=9$ Dirac matrices and $\sigma^{1}, \sigma^{2}$ and $\sigma^{3}$ the Pauli matrices. These transformation rules are derived along the same line as described in section 6 . In what follows, we will perform a dimensional reduction on the action of $D=11$ supergravity on two tori $S^{1} \times S^{1}$ to confirm the coupling in (8.2), but we will not touch the SUSY transformation rules in eq. (8.3) and leave them as a prediction.

In order to have

$$
\sqrt{-g_{11}} R_{11}=\sqrt{-g_{9}} R_{9}+\cdots
$$

the relation between the $D=11$ metric $g_{11}$ and the $D=9$ canonical metric $g_{9}$ has to be

$$
g_{11}=\left(\begin{array}{ccc}
\varphi^{-\frac{2}{7}} g_{9} & & \\
& \varphi & \\
& & \varphi
\end{array}\right)
$$

where $R_{11}$ and $R_{9}$ are $D=11$ and $D=9$ Ricci scalars, respectively, and the $\varphi$ is essentially the dilaton in (8.2). Using (8.7), the precise relation of (8.6) is

$$
\sqrt{-g_{11}} R_{11}=\sqrt{-g_{11}}\left[R_{9}-\frac{9}{2 \cdot 7}\left(\frac{\partial \varphi}{\varphi}\right)^{2}\right]
$$

In order to compare (8.8) with (8.2), we have to take the kinetic term of the dilaton field with standard normalization factor $1 / 2$, which is achieved by setting

$$
\varphi=e^{-\frac{\sqrt{7}}{3} \phi}
$$

By using (8.7) and (8.9), it is easy to show that

$$
\sqrt{-g_{11}} F_{4}^{2}(11) \rightarrow e^{-\frac{2}{\sqrt{7}} \phi} \sqrt{-g_{9}} F_{4}^{2}(9)
$$

where $F_{4}^{2}(11)$ is the square of the rank-4 antisymmetric tensor of $D=11$ supergravity, and $F_{4}^{2}(9)$ is the correspondence of $D=9, N=2$ supergravity. It is easy to see that our prediction of the coupling in (8.2) is confirmed. 


\section{Conclusion}

We have made it clear when and why the dilaton-antisymmetric tensor couplings in the supergravity theories can be determined by our approach. The most important feature of this approach is the equal on-shell matching of bosonic and fermionic degrees of freedom, which implies supersymmetries both on space-time and on world-volume through the socalled $\kappa$-symmetry. Therefore, we should not be so surprised by the determined couplings since they are determined in supergravity theories by supersymmetry, too. Our results indicate also that there might exist fundamental Type II $p$-branes. The known actions of the Type I super $p$-branes may provide a starting point toward constructing those of the recently classified Type II $p$-branes [9, 14]. This paper provides just a primary step to spell out supergravity theories in the sense that we have considered only the simplest field configurations. We expect more, if sophisticated supersymmetric field configurations are considered. In turn, we may also benefit from this for understanding the Type II $p$-branes better.

\section{Acknowledgements}

The author would like to express thanks to M. J. Duff, C. Kounnas and F. Quevedo for discussions, and to P. Howe for the help on understanding the relation between the equations of motion and the superspace constraints in supergravity theories. 


\section{References}

[1] A. Dabholkar, G. W. Gibbons, J. A. Harvey and F. Ruiz Ruiz, Nucl. Phys. B340 (1990) 33.

[2] M. J. Duff and J. X. Lu, Nucl. Phys. B354 (1991) 141.

[3] M. J. Duff and K. Stelle, Phys. Lett. B253 (1991) 113.

[4] C. Callan, J. Harvey and A. Strominger, Nucl. Phys. B367 (1991) 60.

[5] M. J. Duff and J. X. Lu, Phys. Lett. B273 (1991) 409.

[6] G. Horowitz and A. Strominger, Nucl. Phys. B360 (1991) 197.

[7] A. Sen, Phys. Lett. B274 (1992) 34.

[8] S. F. Hassan and A. Sen, Nucl. Phys. B375 (1992) 103.

[9] M. J. Duff and J. X. Lu, "Black and super $p$-branes in diverse dimensions", CERNTH.6675/92 and CTP-TAMU-54/92 (1992).

[10] J. Strathdee, Int. J. Mod. Phys. A2 (1987) 273.

[11] A. Salam and E. Sezgin, "Supergravities in Diverse Dimensions", North-Holland and World Scientific.

[12] A. Achucarro, J. Evans, P. Townsend and D. Wiltshire, Phys. Lett. B198 (1987) 441.

[13] E. Bergshoeff, E. Sezgin and P. Townsend, Phys. Lett. B189 (1987) 75.

[14] M. J. Duff and J. X. Lu, "Type II p-branes: The Brane-Scan Revisited", CERNTH.6560/92 and CTP/TAMU-37/92 (to appear in Nucl. Phys. B).

[15] J. X. Lu, "Supersymmetric Extended Objects", Texas A\&M, Ph.D. Thesis (1992).

[16] M. J. Duff, P. S. Howe, T. Inami and K. Stelle, Phys. Lett. B191 (1987) 70.

[17] M. J. Duff and J. X. Lu, "The self-dual Type IIB superstring" (unpublished).

[18] A. Salam and E. Sezgin, Nucl. Phys. B258 (1985) 284. 